\title{
Suspended Microsystems for In-situ TEM Studies of Processes in Gases and Liquids
}

\author{
Sardar Bilal Alam ${ }^{1}$, Eric Jensen ${ }^{1}$, Frances M Ross ${ }^{2}$, Ole Hansen ${ }^{1,4}$, Andy Burrows ${ }^{3}$, Kristian Mølhave ${ }^{1}$ \\ 1. DTU-Nanotech, Dept. of Micro and Nanotechnology, Technical University of Denmark, Denmark \\ 2. IBM Research Division, T. J. Watson Research Center, Yorktown Heights, New York, USA \\ 3. DTU CEN, Center for Electron Nanoscopy, Technical University of Denmark, Denmark \\ 4. CINF-Center for Individual Nanoparticle Functionality, Technical University of Denmark, Denmark
}

Techniques allowing real-time high resolution imaging of nanoscale processes by in-situ TEM are currently under rapid development[1]. Gas phase studies have traditionally been performed in the environmental TEM (ETEM)[2], and recently more advanced sample geometries based on microchip systems have enabled real-time imaging of controlled processes to be combined with, for instance, correlated electrical measurements[3-4]. As well as gas phase studies, microchip based systems also allow imaging of processes in liquids with good resolution [5-8]. Here we report advances in TEM studies of nanowires grown between suspended joule heated microcantilevers and on our development of a suspended microchannel system for TEM imaging of liquid samples and processes.

We formed and characterised nanowire devices by growing silicon nanowires in-situ in the TEM using the VLS mechanism [9] from one joule heated microcantilever to an adjacent cantilever, thereby forming a bridge as shown in Fig 1a. Building on our previous experiments[3-4] we studied the details of contact formation when a hot nanowire grows into contact with a similarly heated adjacent cantilever. An example is shown in Fig 1b, where a silicon to silicon junction forms as the catalytic gold diffuses away. The suspended microcantilever system opens up novel ways to create electrically contacted nanowire devices [10] in addition to allowing in-situ TEM studies of the many simultaneous processes taking place upon contact formation.

To study liquids encapsulated between membrane windows on $m$ icrochips, the reported microchip devices today all involve two bonded chips with thin membranes of silicon dioxide or nitride [11]. To allow better control of the encapsulated volume and geometry, we are exploring devices using monolithic chips with suspended microfluidic channels made from silicon nitride. On the channels thinned sections serve as windows allowing a higher resolution than in the supporting part of the channel. Figure $2 \mathrm{a}$ shows a sketch of such a microchip system and Fig $2 \mathrm{~b}$ presents a first demonstration of the system with a liquid sample mixed with microscopic gas bubbles in a $1 \mu \mathrm{m}$ high and $4 \mu \mathrm{m}$ wide channel. The system's novel geometry is expected to improve the imaging resolution by providing precise control over the channel height and the possibility of achieving small regions with ultrathin membranes for the best possible resolution. 

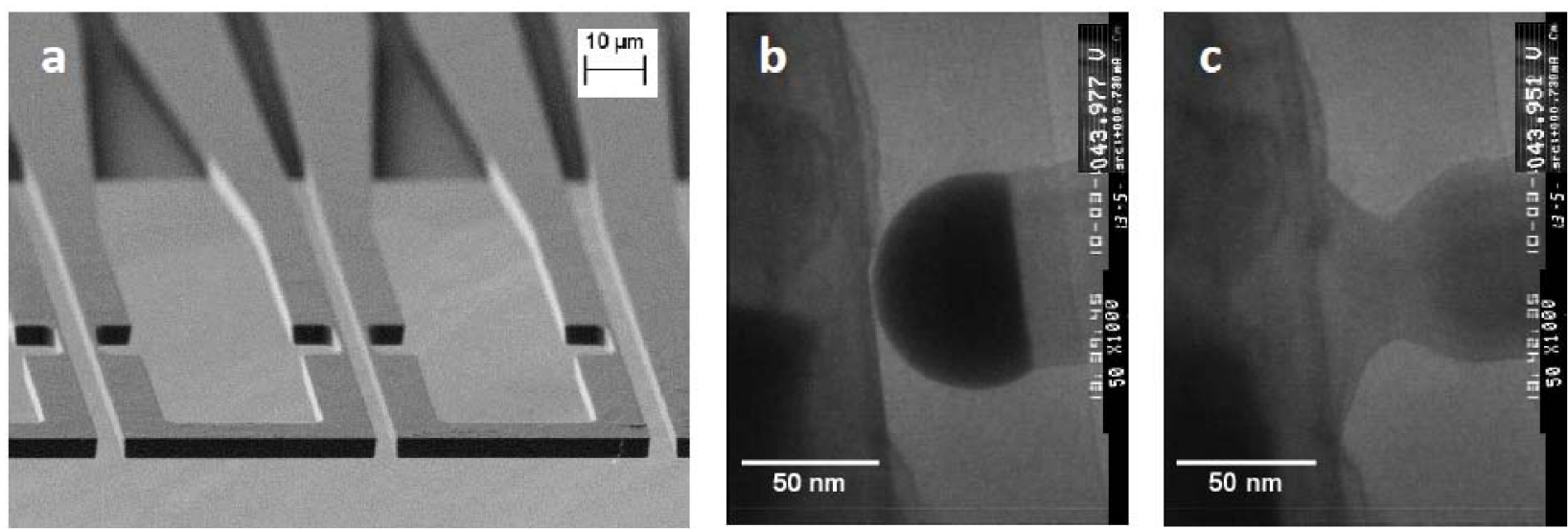

Figure 1. (a) SEM image of suspended monocrystalline silicon cantilevers. Joule heating of adjacent cantilevers makes in-situ TEM VLS growth possible in disilane gas using eutectic particles formed from a thin film of gold deposited onto the cantilevers. (b) TEM image of a silicon nanowire about to reach the side wall of an adjacent heated cantilever, and, (c) the subsequent formation of a silicon-silicon junction as the catalytic gold diffuses away.
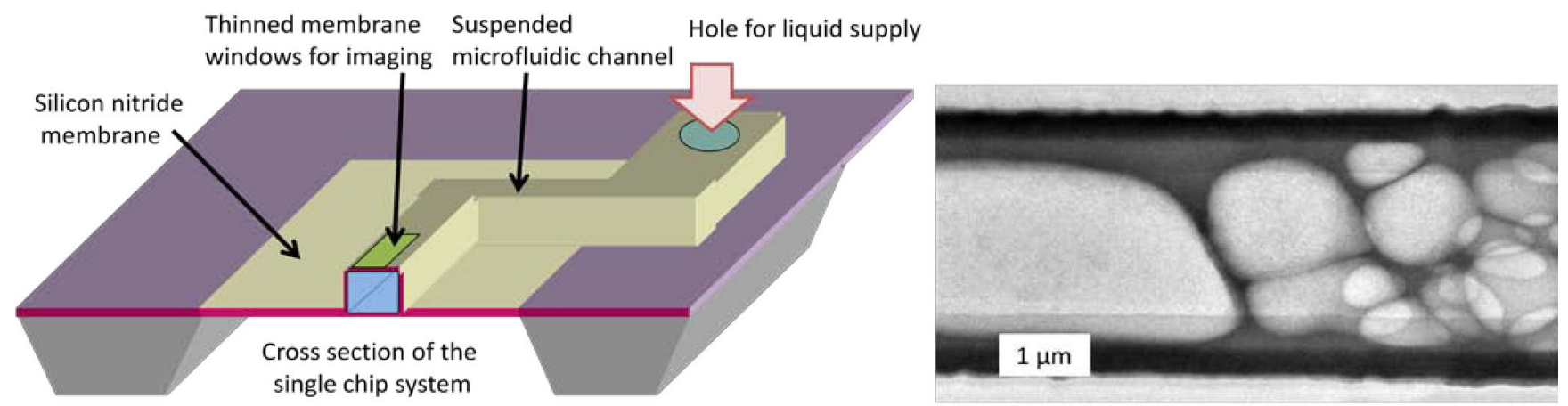

Figure 2. Schematic cross section of the suspended microfluidic channel system for liquid phase TEM imaging. The silicon nitride microfluidic channel crosses a silicon nitride membrane providing mechanically stable suspension. Thinned regions in the channel allow locally improved resolution. The composite overview TEM image shows a mixture of air bubbles and water in a channel.

References:

[1] F. (Feng) Tao et al., Science, vol. 331, Jan. 2011, pp. $171-174$.

[2] J.B. Wagner et al., Micron, vol. 43, Nov. 2012, pp. 1169-1175.

[3] C. Kallesøe et al., Small, vol. 6, 2010, pp. 2058-2064.

[4] C. Kallesøe et al., Nano Letters, vol. 12, Jun. 2012, pp. 2965-2970.

[5] M.J. Williamson et al., Nature Materials, vol. 2, 2003, pp. 532-536.

[6] A. Radisic et al., The Journal of Physical Chemistry B, vol. 110, Apr. 2006, pp. 7862-7868.

[7] D. Li et al., Science, vol. 336, May. 2012, pp. 1014-1018.

[8] H.-G. Liao et al., Science, vol. 336, May. 2012, pp. 1011-1014.

[9] F.M. Ross, Reports on Progress in Physics, vol. 73, Nov. 2010.

[10] M. Islam et al., Nanotechnology, vol. 15, May. 2004, pp. L5-L8.

[11] N. de Jonge et al., Nature Nanotechnology, vol. 6, Oct. 2011, pp. 695-704.

[12] The authors acknowledge the assistance of M. C. Reuter and A. W. Ellis of IBM and funding from FTP Case No. 10-082797 Nanolive, DFF- Sapere Aude LiquidEM, The Danish National Research Foundation's Center for Individual Nanoparticle Functionality (DNRF54), and DTU CEN. 